\title{
Computational Challenge of Fractional Differential Equations and the Potential Solutions: A Survey
}

\author{
Chunye Gong, ${ }^{1,2,3}$ Weimin Bao, ${ }^{1,2}$ Guojian Tang, ${ }^{1}$ Yuewen Jiang, ${ }^{4}$ and Jie Liu ${ }^{3}$ \\ ${ }^{1}$ College of Aerospace Science and Engineering, National University of Defense Technology, Changsha 410073, China \\ ${ }^{2}$ Science and Technology on Space Physics Laboratory, Beijing 100076, China \\ ${ }^{3}$ School of Computer Science, National University of Defense Technology, Changsha 410073, China \\ ${ }^{4}$ Department of Engineering Science, University of Oxford, Oxford OX2 OES, UK
}

Correspondence should be addressed to Chunye Gong; gongchunye@gmail.com

Received 13 June 2014; Revised 6 August 2014; Accepted 9 September 2014

Academic Editor: Guido Maione

Copyright (C) 2015 Chunye Gong et al. This is an open access article distributed under the Creative Commons Attribution License, which permits unrestricted use, distribution, and reproduction in any medium, provided the original work is properly cited.

\begin{abstract}
We present a survey of fractional differential equations and in particular of the computational cost for their numerical solutions from the view of computer science. The computational complexities of time fractional, space fractional, and space-time fractional equations are $O\left(N^{2} M\right), O\left(N M^{2}\right)$, and $O(N M(M+N))$ compared with $O(M N)$ for the classical partial differential equations with finite difference methods, where $M, N$ are the number of space grid points and time steps. The potential solutions for this challenge include, but are not limited to, parallel computing, memory access optimization (fractional precomputing operator), short memory principle, fast Fourier transform (FFT) based solutions, alternating direction implicit method, multigrid method, and preconditioner technology. The relationships of these solutions for both space fractional derivative and time fractional derivative are discussed. The authors pointed out that the technologies of parallel computing should be regarded as a basic method to overcome this challenge, and some attention should be paid to the fractional killer applications, high performance iteration methods, high order schemes, and Monte Carlo methods. Since the computation of fractional equations with high dimension and variable order is even heavier, the researchers from the area of mathematics and computer science have opportunity to invent cornerstones in the area of fractional calculus.
\end{abstract}

\section{Introduction}

The idea of fractional is natural. If $\partial u / \partial x$ and $\partial^{2} u / \partial x^{2}$ exist, $\partial^{1.5} u / \partial x^{1.5}$ maybe exists too. Fractional equations can be used to describe some physical phenomena more accurately than the classical integer order differential equation [1]. Fractional differential equations (FDEs) provide a powerful instrument for the description of memory and hereditary properties of different substances. The fractional diffusion equations play an important role in dynamical systems of semiconductor research, hydrogeology, bioinformatics, finance [2], and other research areas [3-6]. Rajeev and Kushwaha [7] presented a mathematical model describing the time fractional anomalous diffusion process of a generalized Stefan problem which is a limiting case of a shoreline problem. Space fractional advection-diffusion equations arise when velocity variations are heavy tailed and describe particle motion that account for variation in the flow field over the entire system [8]. FDEs may be divided into two fundamental types: time fractional differential equations and space fractional differential equations. For the fractional ordinary equations and fractional order control systems are also studied $[9,10]$. The stability of fractional order control systems attracts many attentions [11, 12]. For example, Laguerre continued fraction expansion of the Tustin fractional discrete-time operator was investigated by Maione [13].

Some analytical methods were proposed for fractional differential equation $[14,15]$. Saha Ray [16] presented the analytical solutions of the space fractional diffusion equations by two-step Adomian decomposition method. Momani and Odibat [17] gave a comparison between the homotopy perturbation method and the variational iteration method for linear fractional partial differential equations. By using initial conditions, the explicit solutions of the equations have 
been presented in the closed form and then their solutions have been represented graphically. Because most of fractional problems cannot be solved analytically, more and more works focus on their numerical solutions. There are many numerical solutions proposed for fractional equations [18], such as finite difference method (FDM) [18]. FDM is intuitive to understand and easy to learn for inexperienced researcher from the areas rather than mathematics. So this survey focuses on FDM for fractional equations.

For the numerical solutions of different differential equations, the area of mathematics pays much attention to approximating the equation more accurately and faster (accuracy and speed). The area of computer science mainly focuses on the runtime (speed) and code reuse (software). The numerical methods (mathematic area) have eternal value and may exist along with the existence of human culture [19-21]. The implementations (computer area) are closer to the human society and the real applications but vary quickly along with the computer architecture [22-28]. The computational cost of the numerical solutions for fractional equations is much heavier than that for the traditional integer order equations. In the near future, the fractional problems with high dimension, long time iterations, and huge grid points will need to be solved. These problems are real challenge for today's computer technologies and algorithms.

\section{Fractional Differential Equations}

2.1. Origins. In 1695, L'Hopital wrote to Leibniz asking him about a particular notation he had used in his publications for the $n$ th-derivative of the linear function $f(x)=x, D^{n} x / D x^{n}$ $[29,30]$. L'Hopital's posed the question to Leibniz: what would the result be if $n=1 / 2$ ? Leibniz's response was "An apparent paradox, from which one day useful consequences will be drawn." In these words fractional calculus was born [31]. Later, Fourier, Euler, and Laplace dabbled with fractional calculus [32].

2.2. A Short Summary of FDE. There are mainly three kinds of FDEs:

(1) time fractional $[33,34]$,

(2) space fractional [35],

(3) space-time fractional [36].

One of the main challenges in fractional differential equation is the nonlocality of the fractional operator. In the case of a time fractional derivative, one needs to store all the history, whereas in the case of a space fractional derivative, one needs to deal with almost-dense matrices.

The partial differential equations (PDEs) mainly have three categories: parabolic, hyperbolic, and elliptic. There are corresponding FDEs to deal with the fractional conditions. There are various FDEs listed below:

(1) the parabolic, widely studied fractional diffusion equation [37, 38],

(2) the hyperbolic Telegraph equation [39],
(3) the elliptic fractional Laplace equation [40],

(4) fractional Black-Scholes equation in computational finance [41],

(5) fractional wave equation for sound, light, and water waves [42],

(6) fractional Fokker-Planck equations describing the time evolution of the probability density function of the velocity of a particle [43],

(7) fractional Euler equations and fractional NavierStokes equations [44] for fluid dynamics [45],

(8) fractional kinetic equations for motion of objects [46],

(9) the perfect, fractional Maxwell's equations for electrodynamics [47],

(10) fractional Boltzmann equations for particle transport [48].

We can suppose that if there is a PDE, there will be a FDE. The reason is that replacing the integer derivative with fractional derivative and solving the FDE with different numerical methods is not a hard job. below:

The numerical schemes [49] for these FDEs are listed

(1) finite difference method (FDM) [18, 50, 51],

(2) finite element method [52-54],

(3) finite volume method [55-57],

(4) Adomian decomposition method [58],

(5) Fourier transform method [59],

(6) spectral method [60],

(7) meshless method [61-63],

(8) exponential difference method $[64,65]$,

(9) Monte Carlo method [66].

The Monte Carlo (MC) method, which uses repeated random sampling to obtain numerical results, belongs to undetermined computational methods.

2.3. A Hot Topic in Recent Years. Machado et al. [29] collected 20 special issues on fractional calculus. In 2011 and 2012, there are about 7 special issues on this topic. There are more special issues in the nearby four years (2011-2014) than those of 1999 to 2010 [29]. And there are additional 20 special issues in the years of 2013 and 2014. So the researches on fractional calculus can be regarded as a collective revelry.

\section{Computational Challenge}

\subsection{A Classical Partial Differential Equation (PDE)}

3.1.1. Heat Equation/Diffusion Equation. The heat equation is a parabolic PDE which describes the distribution of heat (or variation in temperature) in a given region over time, 
shown in (1). This equation is also known as the diffusion equation:

$$
\begin{aligned}
& \frac{\partial u(x, t)}{\partial t}=d(x) \frac{\partial^{2} u(x, t)}{\partial x^{2}}+f(x, t), \\
& u(x, 0)=\phi(x), \quad x \in\left[x_{L}, x_{R}\right], \\
& u\left(x_{L}, t\right)=\varphi(t), \quad t \in[0, T], \\
& u\left(x_{R}, t\right)=\psi(t), \quad t \in[0, T],
\end{aligned}
$$

where the $d(x)$ is the diffusion constant.

3.1.2. Numerical Method. Define $t_{n}=n \tau, x_{i}=$ ih for $0 \leq n \leq$ $N, 0 \leq i \leq M$, where $M$ and $N$ are positive integer and $\tau=$ $T / N, h=\left(x_{R}-x_{L}\right) / M$ are time step size and space step size, respectively. Define $u_{i}^{n}, f_{i}^{n}, d_{i}$ as the numerical approximation to $u\left(x_{i}, t_{n}\right), f\left(x_{i}, t_{n}\right)$, and $d\left(x_{i}\right)$. Using a forward difference at time $t_{n}$ and a second-order central difference for the space derivative at position $x_{i}$, we get the explicit finite difference approximation for the one-dimensional heat equation:

$$
\frac{u_{i}^{n+1}-u_{i}^{n}}{\tau}=d_{i} \frac{u_{i+1}^{n}-2 u_{i}^{n}+u_{i-1}^{n}}{h^{2}}+f_{i}^{n}
$$

The $u_{i}^{n+1}$ can be obtained by this way:

$$
u_{i}^{n+1}=a_{1} u_{i+1}^{n}+a_{2} u_{i}^{n}+a_{3} u_{i-1}^{n}+\tau f_{i}^{n}
$$

where $a_{1}=a_{3}=-d_{i} \tau / h^{2}$ and $a_{2}=1-2 d_{i} \tau / h^{2}$.

If we use the backward difference at time $t_{n+1}$ and a second-order central difference for the space derivative at position $x_{i}$ we get the explicit finite difference approximation for (1):

$$
\frac{u_{i}^{n+1}-u_{i}^{n}}{\tau}=d_{i} \frac{u_{i+1}^{n+1}-2 u_{i}^{n+1}+u_{i-1}^{n+1}}{h^{2}}+f_{i}^{n+1} .
$$

The $u_{i}^{n+1}$ can be obtained by this way:

$$
a_{1} u_{i+1}^{n+1}+a_{2} u_{i}^{n+1}+a_{3} u_{i-1}^{n+1}=u_{i}^{n}+\tau f_{i}^{n},
$$

where $a_{1}=a_{3}=d_{i} \tau / h^{2}$ and $a_{2}=1+2 d_{i} \tau / h^{2}$.

The classical PDE is used to compare the fractional equations. For convenience, there are several hypotheses for this paper.

(1) We just focus on the explicit FDM of (3), because these kinds of comparisons between fractional equations and classical equations are straightforward. The comparison between implicit FDE of (5) and implicit schemes of fractional equations is more complex [67].

(2) The computational cost of diffusion coefficient $d_{i}$ and source $f_{i}^{n}$ (or $\left.f_{i}^{n+1}\right)$ is ignored, because they only need compute once. And they are different with different equations and make the comparison complicated.
3.1.3. Computational Cost. Each grid point of time step $t_{n+1}$ needs 4 multiplications and 3 additions. There are $M-1$ grid points in each time step. So each time step needs $7(M-1)$ arithmetic logic operations. There are about $N$ time steps. So the total computational cost is about $7 N(M-1)$. The computational cost will vary linearly along the number of time steps and grid points.

\subsection{Time Fractional Diffusion Equation}

3.2.1. Numerical Method. Liu et al. [63] developed an implicit radial basis function ( $\mathrm{RBF}$ ) meshless approach for time fractional diffusion equations and found that the presented meshless formulation is very effective for modeling and simulation of fractional differential equations. Murillo and Yuste developed an explicit difference method for solving fractional diffusion with Caputo form [68]:

$$
\begin{aligned}
& \frac{\partial^{\alpha} u(x, t)}{\partial^{\alpha} t}=\frac{\partial^{2} u(x, t)}{\partial x^{2}} \quad(0<\alpha<1), \\
& u(x, 0)=g(x), \quad x \in\left(0, x_{R}\right), \\
& u(0, t)=u\left(x_{R}, t\right)=0
\end{aligned}
$$

on a finite domain $0<x<x_{R}$ and $0 \leq t \leq T$.

The explicit finite difference approximation for (6) is described as follows [69]:

$$
\tau^{-\alpha} \sum_{i=0}^{n+1} w_{i}\left(u_{j}^{n+1-i}-u_{j}^{0}\right)=\frac{1}{h^{2}}\left(u_{j+1}^{n}-2 u_{j}^{n}+u_{j-1}^{n}\right),
$$

where $w_{i}$ is the normalized Grünwald weight defined with

$$
w_{i}=(-1)^{i}\left(\begin{array}{c}
\alpha \\
i
\end{array}\right)
$$

Define $\mu=\tau^{\alpha} / h^{2}, b_{n}=\sum_{i=0}^{n-1} w_{i}$, and $U^{n}=\left(u_{1}^{n}, u_{2}^{n}, \ldots\right.$, $\left.u_{M-1}^{n}\right)^{T}$; we can get

$$
U^{n+1}=b_{n+1} U^{0}-\sum_{i=1}^{n+1} w_{i} U^{n+1-i}+\mu A U^{n}
$$

where matrix $A$ is a tridiagonal matrix, defined by

$$
A_{M-1 \times M-1}=\left(\begin{array}{ccccc}
-2 & 1 & & & \\
1 & -2 & 1 & & \\
& \bullet & \bullet & & \\
& & \bullet & \bullet & 1 \\
& & & 1 & -2
\end{array}\right) \text {. }
$$

3.2.2. Computational Cost. In order to get $U^{n+1}$, the rightsided computation of (9) should be performed. There are mainly one tridiagonal matrix-vector multiplication, many constant-vector multiplications, and many vector-vector additions in the right-sided computation.

(1) The tridiagonal matrix-vector multiplication is $A U^{n}$ and a new vector $U^{n+1}=A U^{n}$ is got. 
(2) The constant-vector multiplications are $V^{\prime}=b_{n+1} U^{0}$, $V^{\prime \prime}=\mu V^{1}, V^{i}=w_{i} U^{n+1-i}$.

(3) The vector-vector additions are $U^{n+1}=U^{n+1}+V^{\prime}+$ $V^{\prime \prime}-\sum_{i=1}^{n+1} V^{i}$

There are about $5(M-1)$ operations for tridiagonal matrix-vector multiplication. For time step $t_{n+1}$ needs $(n+$ $8)(M-1)$ arithmetic logic operations with $n=1 \rightarrow N$. So the total computational cost for (9) is about

$$
\begin{aligned}
8 N(M-1)+(1+2+\cdots+N-1)(M-1) \\
\quad=8 N(M-1)+\frac{N(N-1)(M-1)}{2} \\
\quad=N\left(\frac{N}{2}+7.5\right)(M-1) .
\end{aligned}
$$

The computational cost varies linearly along the number of grid points but squares with the number of time steps.

\subsection{Space Fractional Diffusion Equation}

3.3.1. Numerical Method. A classical numerical scheme for the space fractional diffusion equation is the second-order fractional Crank-Nicolson method proposed by Tadjeran et al. $[38,70]$, where the Richardson extrapolation technique is used to the first order shift Grünwald formula for space fractional derivative. Tadjeran et al. [70] presented a practical numerical method in time and space to solve a class of initialboundary value with variable coefficients on a finite domain for

$$
\frac{\partial u(x, t)}{\partial t}=d(x) \frac{\partial^{\alpha} u(x, t)}{\partial x^{\alpha}}+q(x, t)
$$

on a finite domain $x_{L}<x<x_{R}$ with $1<\alpha<2$ and $0 \leq t \leq T$. The case of $1<\alpha<2$ models a super-diffusive flow in which a cloud of diffusing particles spreads at a faster rate than the classical diffusion model predictions [71, 72].

The explicit finite difference approximation for (12) is

$$
\frac{u_{i}^{n+1}-u_{i}^{n}}{\tau}=\frac{d_{i}}{h^{\alpha}}\left(\sum_{k=0}^{i+1} g_{\alpha, k} u_{i-k+1}^{n}\right)+q_{i}^{n},
$$

where $u_{i}^{n}=u\left(x_{i}, t_{n}\right), d_{i}=d\left(x_{i}\right)$, and $q_{i}^{n}=q\left(x_{i}, t_{n}\right)$. The $g_{\alpha, k}$ is the normalized Grünwald weight $[70,73]$ :

$$
g_{\alpha, k}=\frac{\Gamma(k-\alpha)}{\Gamma(-\alpha) \Gamma(k+1)} .
$$

These normalized weights only depend on the order $\alpha$ and the index $k$. The resulting equation can be explicitly solved for $u_{i}^{n+1}$ to give

$$
u_{i}^{n+1}=u_{i}^{n}+\frac{d_{i} \tau}{h^{\alpha}}\left(\sum_{k=0}^{i+1} g_{\alpha, k} u_{i-k+1}^{n}\right)+\tau q_{i}^{n} .
$$

3.3.2. Computational Cost. The $i$ th grid point of time step $t_{n+1}$ needs $i+6$ multiplication, addition, and division. There are $M-1$ grid points in each time step. So each time step needs $(M-1) 6+(1+2+\cdots+M-1)=(M-1)((M / 2)+6)$ arithmetic logic operations. There are about $N$ time steps. So the total computational cost is about $N(M-1)((M / 2)+6)$.

\subsection{Riesz Space Fractional Diffusion Equation}

3.4.1. Numerical Method. Shen et al. [74] investigated the Green function and a discrete random walk model for Riesz fractional advection-dispersion equation on infinite domain with an initial condition and also presented implicit and explicit finite differences to this problem on a finite domain. Çelik and Duman [75] applied the Crank-Nicolson method to a fractional diffusion equation which has the Riesz fractional derivative and obtained that the method is unconditionally stable and convergent. The Riesz space fractional reactiondiffusion equation $[76,77]$ is

$$
\begin{aligned}
& \frac{\partial u(x, t)}{\partial t}=-u(x, t)+{ }_{x} D_{0}^{\alpha} u(x, t)+q(x, t), \\
& u(x, 0)=g(x), \quad x \in\left(x_{L}, x_{R}\right), \\
& u\left(x_{L}, t\right)=u\left(x_{R}, t\right)=0
\end{aligned}
$$

with $1<\alpha \leq 2$ and $0 \leq t \leq T$. Both $u(x, t)$ and $g(x)$ are real valued and sufficiently well-behaved function. ${ }_{x} D_{0}^{\alpha} u(x, t)$ is the Riesz space fractional derivative.

With adopting an Euler approximation in time, the explicit difference approximation can be got:

$$
\begin{aligned}
\frac{u_{i}^{n+1}-u_{i}^{n}}{\tau}= & -u_{i}^{n}-\frac{1}{c h^{\alpha}} \\
& \times\left[\sum_{k=0}^{i+1} g_{k} u_{i+1-k}^{n}+\sum_{k=0}^{M-i+1} g_{k} u_{i-1+k}^{n}\right]+f_{i}^{n},
\end{aligned}
$$

where $g_{0}=1, g_{k}=(-1)^{k} \alpha(\alpha-1) \cdots(\alpha-k+1) / k !, k=1,2$, $3, \ldots$ is the normalized Grünwald weight, $c=1 / 2 \cos (\alpha \pi / 2)$, and $f_{i}^{n}=q\left(x_{i}, t_{n}\right)$.

Equation (17) results in a linear system of equations

$$
U^{n+1}=A U^{n}+Q^{n}
$$

where $U^{n}=\left(u_{1}^{n}, u_{2}^{n}, \ldots, u_{M-1}^{n}\right)^{T}, Q^{n}=\left(\tau f_{1}^{n}, \tau f_{2}^{n}, \ldots, \tau f_{M-1}^{n}\right)^{T}$ with $q_{i}^{n}=\tau f_{i}^{n}(1 \leq i<M-1)$, and $A=\left(a_{i j}\right)_{(M-1) \times(M-1)}$ is a matrix of coefficient. $A$ is defined by

$$
a_{i, j}= \begin{cases}-\frac{\tau}{c h^{\alpha}} g_{i+1-j}, & \text { for } 1 \leq j<i-1, \\ -\frac{\tau}{c h^{\alpha}}\left(g_{0}+g_{2}\right), & \text { for } j=i-1, \\ (1-\tau)-2 \frac{\tau}{c h^{\alpha}} g_{1}, & \text { for } j=i, \\ -\frac{\tau}{c h^{\alpha}}\left(g_{0}+g_{2}\right), & \text { for } j=i+1, \\ -\frac{\tau}{c h^{\alpha}} g_{j-i+1}, & \text { for } i+1<j<M-1 .\end{cases}
$$


3.4.2. Computational Cost. From (18), the $M-1$ results are produced by $A U^{n}$ for each time step. The inner product of vectors $v^{1}$ and $v^{2}$ with size $m$ has $m$ multiplications and $m-1$ additions. $A U^{n}$ for each time step needs $(M-1)(2 M-3)$ operations. Assuming $\tau f_{i}^{n}$ is pre-performed, each time step needs $(M-1)(2 M-2)$ arithmetic logic operations. There are about $N$ time steps. So the total computational cost is about $N(M-1)(2 M-2)$. The computational cost will vary linearly along the number of time steps but square with the number of grid points. From analytical view, the computation of (18) is about four times heavier than that of (15).

\subsection{Space-Time Riesz-Caputo Fractional Convection-Diffusion} Equation. The fractional advection-diffusion (dispersion) equation has been applied to many problems. Fractional advection-dispersion equations are used in groundwater hydrology to model the transport of passive tracers carried out by fluid flow in a porous medium [78]. Shen et al. [79] presented an explicit difference approximation and an implicit difference approximation for the space-time Riesz-Caputo fractional advection-diffusion equation with initial and boundary conditions in a finite domain. They proved that the implicit difference approximation is unconditionally stable and convergent, but the explicit difference approximation is conditionally stable and convergent. Liu et al. [80] proposed an implicit difference method and an explicit difference method to solve the space-time fractional advection-diffusion equation and discussed the stability and convergence of the method.

3.5.1. Numerical Method. We consider the following spacetime Riesz-Caputo fractional advection-diffusion equation (STRCFADE) studied by Shen et al. [79]. This equation is obtained by replacing the space-derivative in the advectiondiffusion equation with a generalized derivative of order $\beta_{1}$, $\beta_{2}$ with $0<\beta_{1}<1,1<\beta_{2}<2$ and time-derivative with a generalized derivative of order $\alpha$ with $0<\alpha<1$. We consider

$$
\begin{gathered}
{ }_{0} D_{t}^{\alpha} u(x, t)=B_{1} \frac{\partial^{\beta_{1}} u(x, t)}{\partial|x|^{\beta_{1}}}+B_{2} \frac{\partial^{\beta_{2}} u(x, t)}{\partial|x|^{\beta_{2}}}+f(x, t), \\
u(x, 0)=\phi(x), \quad x \in\left[0, x_{R}\right] \\
u(0, t)=u\left(x_{R}, t\right)=0, \quad t \in[0, T]
\end{gathered}
$$

on a finite domain $0 \leq x \leq x_{R}$ and $0 \leq t \leq T$. The coefficients $B_{1}$ and $B_{2}$ are both positive constants and represent the diffusion (dispersion) coefficient and the average fluid velocity. The $\partial^{\beta_{1}} u(x, t) / \partial|x|^{\beta_{1}}$ and $\partial^{\beta_{2}} u(x, t) / \partial|x|^{\beta_{2}}$ are Riesz space fractional derivatives of order $\beta_{1}$ and $\beta_{2}$, respectively. Then we can get the explicit finite differential approximation for (20) [79]:

$$
\begin{aligned}
u_{i}^{n+1}= & \sum_{j=0}^{n-1}\left(b_{j}-b_{j+1}\right) u_{i}^{n-j}+b_{n} u_{i}^{0}+B_{1} \mu_{1}\left(\sum_{k=-i}^{N-i} \omega_{k}^{\beta_{1}} u_{i+k}^{n}\right) \\
& +B_{2} \mu_{2}\left(\sum_{k=-i}^{N-i} \omega_{k}^{\beta_{2}} u_{i+k}^{n}\right)+\mu_{0} \varphi_{i}^{n}, \quad i=1, \ldots, N-1,
\end{aligned}
$$

$$
\begin{gathered}
u_{0}^{n+1}=u_{N}^{n+1}=0, \\
u_{i}^{0}=\phi_{i}
\end{gathered}
$$

where $\mu_{0}=\tau^{\alpha} \Gamma(2-\alpha), \mu_{1}=\mu_{0} / h^{\beta_{1}}$, and $\mu_{2}=\mu_{0} / h^{\beta_{2}}$. More information can be referred to in [79].

3.5.2. Computational Cost. From Section 3.4.2, we know that the computational cost of $B_{1} \mu_{1}\left(\sum_{k=-i}^{N-i} \omega_{k}^{\beta_{1}} u_{i+k}^{n}\right)+$ $B_{2} \mu_{2}\left(\sum_{k=-i}^{N-i} \omega_{k}^{\beta_{2}} u_{i+k}^{n}\right)+\mu_{0} \varphi_{i}^{n}$ of $N$ time steps is about $N(M-1)(2 M-2)$ operations. From Section 3.2.2, we know that the computational cost of $\sum_{j=0}^{n-1}\left(b_{j}-b_{j+1}\right) u_{i}^{n-j}+b_{n} u_{i}^{0}$ of $N$ time steps with $n$ ranging from 1 to $N$ is about $N(N / 2)(M-1)$. So the total computational cost is about $N(M-1)(2 M-2+(N / 2))$. The computational cost varies quadratically with the number of time steps or the number of grid points.

For fixed $M=4097$, the comparison between the computational costs of the numerical solution among classical PDE, time fractional, space fractional, Riesz space fractional, and space-time Riesz-Caputo fractional equations is shown in Figure 1. For fixed $N=2048$, the computational cost is shown in Figure 2.

3.6. More Challenges. There are more computational challenges listed below:

(1) high dimensional problems [55, 81],

(2) implicit schemes [67, 82],

(3) high order schemes [33, 83],

(4) variable order problems [84],

(5) huge memory space requirement.

The high dimensional problems are more computation expensive $[55,81,85,86]$. For example, the two-dimensional time fractional diffusion equation (2D-TFDE) $[67,87]$ is

$$
\begin{gathered}
{ }_{0} D_{t}^{\alpha} u(x, y, t)=a(x, y, t) \frac{\partial^{2} u(x, y, t)}{\partial x^{2}} \\
+a(x, y, t) \frac{\partial^{2} u(x, y, t)}{\partial y^{2}}+f(x, y, t), \\
u(x, y, 0)=\phi(x, y), \quad(x, y) \in \Omega, \\
\left.u(x, y, t)\right|_{\partial \Omega}=0, \quad t \in[0, T],
\end{gathered}
$$

where $\Omega=\left\{(x, y) \mid 0 \leq x \leq L_{1}, 0 \leq y \leq L_{2}, a(x, y, t)>0\right.$, and $b(x, y, t)>0\}$. 


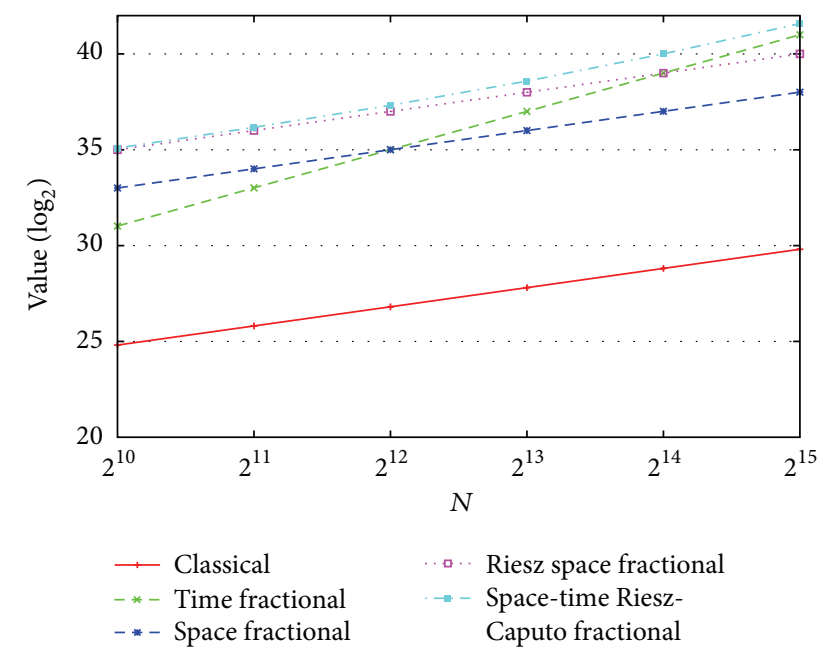

FIGURE 1: Comparison of the computational cost with different grid points for fixed $M=4097$ among classical, time fractional, space fractional, Riesz space fractional, and space-time Riesz-Caputo fractional equations.

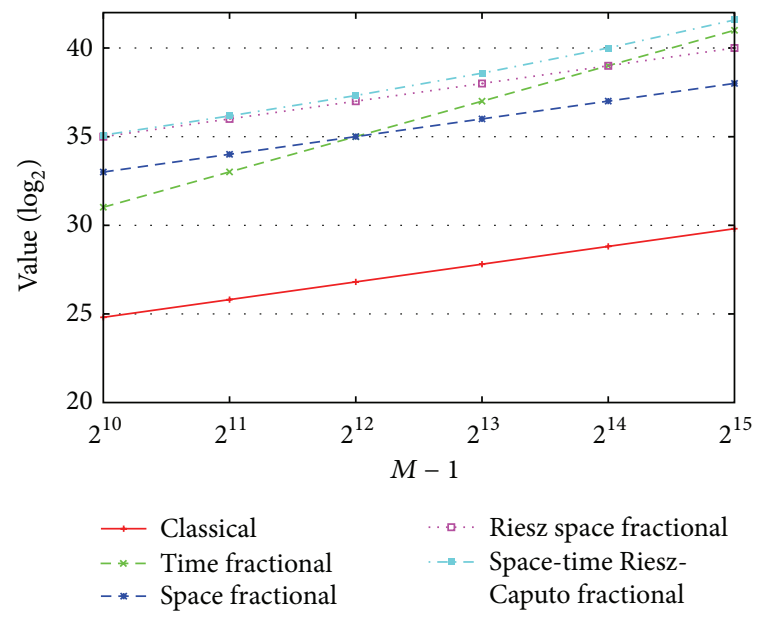

FIGURE 2: Comparison of the computational cost with different time step for fixed $N=2048$ among classical, time fractional, space fractional, Riesz space fractional, and space-time Riesz-Caputo fractional equations.

The approximating scheme is [87]

$$
\begin{aligned}
u_{i, j}^{n+1}- & u_{i, j}^{n}+\sum_{s=1}^{n}\left(b_{s}\right)\left(u_{i, j}^{n+1-s}-u_{i, j}^{n-s}\right) \\
= & \mu_{1} \Gamma(2-\alpha) a_{i, j}^{n}\left(u_{i+1, j}^{n}-2 u_{i, j}^{n}+u_{i-1, j}^{n}\right) \\
& +\mu_{2} \Gamma(2-\alpha) b_{i, j}^{n}\left(u_{i, j+1}^{n}-2 u_{i, j}^{n}+u_{i, j-1}^{n}\right) \\
& +\tau^{\alpha} \Gamma(2-\alpha) f_{i, j}^{n},
\end{aligned}
$$

where $\mu_{1}=\tau^{\alpha} / h_{x}^{2}$ and $\mu_{2}=\tau^{\alpha} / h_{y}^{2}$. The $h_{x}$ and $h_{y}$ are the step size along $X$ and $Y$ directions. The computational complexity is about $O\left(N^{2} M^{2}\right)$, which is much bigger than the computational complexity of one-dimensional problems when the number of grid points is bigger enough.

The direct Gauss elimination for implicit scheme of FDE is not convenient. Solving unsteady FDE with implicit schemes relies on the iteration method at each time step. The variable order problems $[84,88]$ have complex coefficients, which need more arithmetic logic operations. The high order schemes $[33,38,70]$ need more arithmetic logic operations too.

The memory usage belongs to the computing resources. So the huge memory requirement of FDE is a kind of computational challenge in the broader sense. This is especially true for time fractional problems. Ignoring the memory usage of the coefficients and source terms, the one-dimensional equation (9) needs $8(M-1) N$ bytes of memory space and the twodimensional equation (23) needs $8 M^{2} N$ bytes of memory space. For three-dimensional problems, the memory usage is $8 M^{3} N$ at least. It needs $15.625 \mathrm{~PB}\left(1 \mathrm{~PB}=1024^{5}\right.$ bytes $)$ memory space with $M=10240, N=2048$ for three-dimensional problems. Maybe only the most powerful supercomputer [89] can satisfy the huge memory requirement.

\section{Potential Solutions}

There are several ways which can be used to overcome the computational challenge of FDEs.

4.1. Parallel Computing. Large scale applications and algorithms in science and engineering such as neutron transport [90-92], light transport [93], computational fluid dynamics $[94,95]$, molecular dynamics [96], and computational finance and different numerical methods [97] rely on parallel computing $[98,99]$.

Gong et al. [77] present a parallel algorithm for Riesz space fractional diffusion equation based on MPI parallel programming model at the first time. The parallel algorithm is as accurate as the serial algorithm and achieves 79.39\% parallel efficiency compared with 8 processes on a distributed memory cluster system. The parallel implicit iterative algorithm was studied for two-dimensional time fractional problem and a task distribution model is shown in Figure 3 [67]. $M_{x}, M_{y}, P_{x}, P_{y}$ stand for the discrete grid points, parallel processes along $X, Y$ directions.

Domain decomposition method is regarded as the basic mathematical background for many parallel applications [100-102]. A domain decomposition algorithm for time fractional reaction-diffusion equation with implicit finite difference method was proposed [103]. The domain decomposition algorithm keeps the same parallelism as Jacobi iteration but needs much fewer iterations.

Diethelm [104] implemented the fractional version of the second-order Adams-Bashforth-Moulton method on a parallel computer and discussed the precise nature of the parallelization concept. Parallel computing has already appeared in some studies on FDEs, but until today their power for approximating fractional derivatives and solving FDEs has not been fully recognized. 


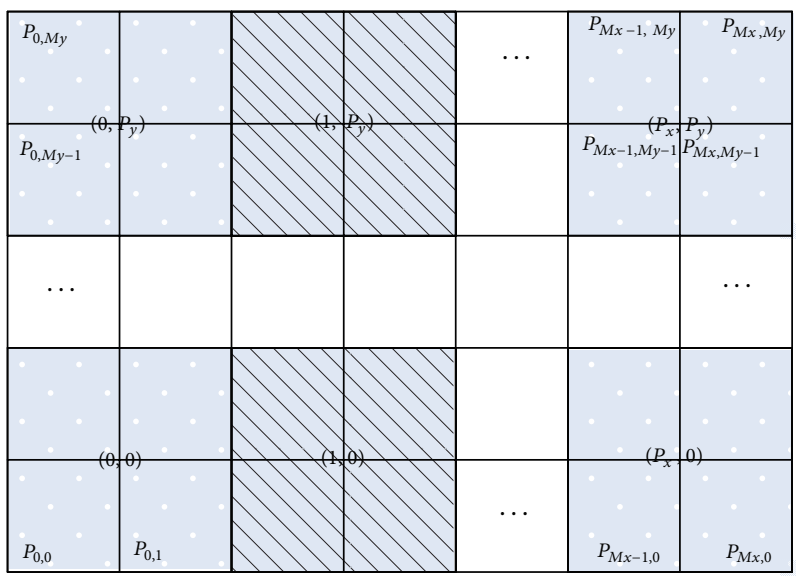

FIGURE 3: The two-dimensional task distribution model for 2DTFDE.

\subsection{Memory Access Optimization (Fractional Precomput-} ing Operator). Memory access optimization is generally regarded as a technology of computer architecture. It is also useful from the view of applications. One feature of the modern computer architecture is multilayer memory. For example, the traditional CPU has fast cache and slow main memory. The new graphics processing unit (GPU) has fast shared memory and slow memory. Reusing the data in shared memory is a key point to improve the performance of GPU applications [105-109]. A very useful optimization is presented for fractional derivative $[69,110]$. We can name this fractional precomputing operator, which will be a very basic and useful optimizing technology for the implementation of fractional algorithms and applications. The example of fractional precomputing operator can refer to Algorithms 4 and 5 in reference [69].

On CPU platform, an optimization of the sum of constant vector multiplication is presented and 2-time speedup can be got for both serial and parallel algorithm for the time fractional equation [69]. The key technology is reusing the data in cache through loop unrolling. Zhang et al. [110] presented code optimization for fractional Adams-Bashforth-Moulton method by loop fusion and loop unrolling.

On GPU platform, Liu et al. [111] presented an optimized CUDA kernel for the numerical solution of time fractional equation and 1.2-2.7-time performance improvement is achieved.

4.3. Short Memory Principle. The short memory principle [3] means the unknown grid points only rely on the recent past (in time) or near neighbors (in space). This principle has been proved to be an easy and powerful way for various kinds of fractional differential equations [112, 113]. The short memory principle is also called fixed memory principle or logarithmic memory principle. The idea of short memory principle is simple. Taking time fractional equation as an example in Section 3.2.1, the value of $w_{i}$ becomes smaller while $i$ is increasing. In (9), the accumulation of $\sum_{i=1}^{n+1} w_{i} U^{n+1-i}$ is replaced with $\sum_{i=\min \{1, n+1-K\}}^{n+1} w_{i} U^{n+1-i} . K$ is a positive integer, which means if $n$ is very big, the computation of the accumulation is fixed.

Based on short memory principle, some principles with better balance of computation speed and computation accuracy are presented. The logarithmic memory principle is developed with a good approximation to the true solution at reasonable computational cost [114]. Another principle is equal-weight memory principle, in which an equal-weight is applied to all past data in history, and the result is reserved instead of being discarded [115]. The equal-weight memory principle is an interesting and useful approximation method to fractional derivative.

4.4. FFT Based Solution. Because of the nonlocal property of fractional differential operators, the numerical methods have full coefficient matrices which require computational cost of $O\left(M^{3}\right)$ for implicit scheme per time step, where $M$ is the number of grid points. Ford and Simpson [114] developed a faster scheme for the calculation of fractional integrals. A reduction in the amount of computational work can be achieved by using a graded mesh, thereby making the $O\left(N^{2}\right)$ method to a $O(N \log N)$ method. The underlying idea is based on the fact that the fractional integral possesses a fundamental scaling property that can be exploited in a natural way [114].

Wang et al. [116, 117] developed a fast finite difference method for fractional diffusion equations, which only requires computational cost of $\mathrm{O}\left(\mathrm{N} \log _{2} N\right)$ while retaining the same accuracy and approximation property as the regular finite difference method. Numerical experiments show that the fast method has a significant reduction of CPU time [86]. The fast method should have a banded coefficient matrix instead of the full matrix. The properties of Toeplitz and circulant matrices, fast Fourier transform (FFT), and inverse FFT are used to reduce the computational cost. The method is also called superfast-preconditioned iterative method for steady-state space fractional diffusion equations [118].

An efficient iteration method for Toeplitz-plus-band triangular systems, which may produced by fractional ordinary differential equations, was developed. Some methods such as matrix splitting, FFT, compress memory storage, and adjustable matrix bandwidth are used in the presented solution. The interesting technologies are the adjustable matrix bandwidth and solving fractional ordinary differential equations with iteration method. The experimental results show that the presented efficient iteration method is 4.25 times faster than the regular solution [10].

4.5. Alternating Direction Implicit Method. The alternating direction implicit (ADI) method is a finite difference method for solving traditional PDEs. The approximation methods for fractional equations result in a very complicated set of equations in multiple dimensions, which are hard to solve [67, $81,85]$. So the ADI method is developed for high dimensional problems [33, 117]. The advantage of the fractional ADI method is that the equations that have to be solved in each step have a simpler structure. The time fractional problems 
can be solved efficiently with the tridiagonal matrix algorithm [33]. The space fractional problems can use the FFT to accelerate the computation [117].

4.6. Multigrid Method. The multigrid method is usually exploited for solving ill-conditioned systems. The main idea of multigrid is to accelerate the convergence of a basic iterative method by global correction form, accomplished by solving a coarse problem. Pang and Sun [119] proposed a multigrid method to solve the initial-boundary value problem of a fractional diffusion equation. The experimental results show that the multigrid + FFT method runs hundred times faster than Gaussian elimination method and the conjugate gradient normal residual (CGNR) method. A full $\mathrm{V}$-cycle multigrid method is proposed for the stationary fractional advection dispersion equation [120] and ten-time performance improvement is achieved.

4.7. Preconditioning Technology. Preconditioning is typically related to reducing a condition number of the problem with iterative methods. It shows that both the average number of iterations and the CPU time by the PCGNR (preconditioner CGNR) method with circulant preconditioners are much less than those by the CGNR method and less than that by the multigrid method [121]. The circulant preconditioner [121], banded preconditioner [122], fast Poisson preconditioners [123], and preconditioned conjugate gradient squared method plus FFT [118] are developed for different FDEs.

4.8. Relationships among These Potential Solutions. The potential solutions for the computational challenge of FDE are investigated above. Many people will be curious about these relationships: can we combine these methods to develop a fastest solution for FDEs? The answer is still unknown. The performance of these solutions varies from different FDE applications. Their relationships are shown in Table 1. The PC, MAO, SMP, FFT, ADI, MGM, and PT stand for parallel computing, memory access optimization, short memory principle, FFT based solution, alternating direction implicit method, multigrid method, and preconditioning technology. The score means the degrees of the two solutions are harmonious. Higher score means using two solutions can achieve better performance.

For time fractional derivative [124], only memory access optimization [69, 111] and short memory principle [3] are useful. Here, the time fractional derivative is different from time fractional problems. For example, the one- and twodimensional FDEs are parallelized by Gong et al. [67, 69]. These parallel algorithms are based on the partition of space not time.

\section{Future Directions}

5.1. Fractional Killer Applications. Killer application is a kind of application that is so necessary or desirable that it proves the core value of some larger technology. A killer application is something like Project Apollo in space technology, the IBM 360 in personal computer industry, and the IPhone in
TABLE 1: Relationships among the potential solutions for space fractional derivative.

\begin{tabular}{lccccccc}
\hline & PC & MAO & SMP & FFT & ADI & MGM & FT \\
\hline PC & - & 3 & 3 & $2^{\mathrm{a}}$ & 3 & $2^{\mathrm{a}}$ & $2^{\mathrm{a}}$ \\
MAO & & - & 3 & $1^{\mathrm{b}}$ & 3 & 3 & 3 \\
SMP & & & - & $1^{\mathrm{c}}$ & 3 & 3 & 3 \\
FFT & & & & - & 3 & 3 & 3 \\
ADI & & & & - & 3 & 3 \\
MGM & & & & & & - & $2^{\mathrm{d}}$ \\
PT & & & & & & & -
\end{tabular}

${ }^{\mathrm{a}}$ The parallel efficiency of FFT, multigrid, preconditioner is limiting.

${ }^{\mathrm{b}}$ It is hard to use MAO within FFT.

${ }^{\mathrm{c}}$ Because of the Toeplitz structure, FFT cannot use short memory principle.

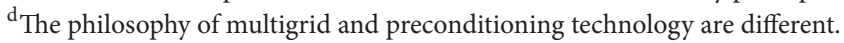
But there are some multigrid preconditioners.

the smart phone industry. The fractional research still lacks these kinds of killer applications. It needs fractional applications to solve scientific or engineering problems in physical world, such as the fractional flow/control of hypersonic vehicle, not only the academical problems. The fractional killer application should be proved that it is more useful than the traditional classical application. Solving real problems in physical world will build an economic foundation for fractional researches.

5.2. Parallel Computing. The technologies of parallel computing should be regarded as a basic method to overcome the computational challenge for FDEs. There are three potential solutions for the computational challenge of FDEs. The short memory principle is an experimental method, which is useful in real fractional applications. The $O(N \log N)$ methods [117] used the property of Toeplitz matrices and FFT technology. Parallel computing is a foundational technology for scientific and engineering computation. Fortunately, the short memory principle and $O(N \log N)$ methods are compatible with parallel computing. So it is interesting to develop algorithms which is faster than $O(N \log N)$ methods. The numerical methods of space fractional equations with global dependence are much harder to be parallelized than that of the time fractional equations. So the task distribution model, load balance of the parallel algorithm for space fractional equations should be paid attention to.

5.3. High Performance Iteration Methods. Different kind of numerical methods is very easily found for FDEs. The direct methods such as Gauss elimination are not suitable for large scale fractional applications. The iterative methods for these numerical methods are not fully studied and very few works can be found $[118,125]$. We think that different iterative methods, such as Jacobi method and Gauss-Seidel and Krylov subspace method, are effective for fractional linear systems which are produced from FDEs. Does the coefficient matrix of FDEs have some other special properties or not? The answer is still unknown. The convergence and stability of these iterative methods should be proved as well. 
5.4. High Order Schemes for Fractional Derivatives. The traditional partial derivatives have many high order schemes. For time fractional equation, the high order schemes for traditional integer derivative are not hard to build. But for fractional derivatives, the high order schemes are under developing [70, 126-128]. High order schemes will be used in the numerical solutions of FDEs where high accuracy is required in the presence of shocks or discontinuities.

5.5. Monte Carlo Method. The Monte Carlo method has advantage in solving nonlinear, high dimensional, complex geometry problems. In order to get the approximation of a small domain, the determined methods, such as FDM, must resolve the total definition domain with boundary conditions. The Monte Carlo method only focuses on the small domain. This property is very useful if we are only interested in this small domain. The Monte Carlo method is easy to be parallelized and needs much less memory space than determined methods. The fractional equations are a kind of nonlinear problem and their high dimensional problems are very computation intensive. So Monte Carlo method for FDEs needs to be studied in the future. Because of the high nonlinear and nonlocal property of FDEs, the sampling efficiency will be the key point of Monte Carlo method for FDEs.

\section{Conclusions}

In this paper, we give a comprehensive review of FDEs and its computational challenge. This kind of challenge will become an incoming problem for the computer industry if the real fractional problems need to be approximated. We reviewed a wide range of computational costs that come from different kinds of fractional equations. While we have collected several potential solutions on this challenge, we believe that the longterm legacy of solutions will allow the real world scientific and engineering applications come true.

\section{Conflict of Interests}

The authors declare that there is no conflict of interests regarding the publication of this paper.

\section{Acknowledgments}

This research work is supported in part by the National Natural Science Foundation of China under Grants no. 61402039, no. 61303265, and no. 60970033, in part by Specialized Research Fund for the Doctoral Program of Higher Education under Grant no. 20114307110001, and in part by 973 Program of China under Grants no. 2014CB430205 and no. 61312701001 . The authors would like to thank the anonymous reviewers for their helpful comments as well.

\section{References}

[1] R. Klages, G. Radons, and I. Sokolov, Anomalous Transport: Foundations and Applications, Wiley-VCH, Weinheim, Germany, 2008.
[2] R. R. Nigmatullin, T. Omay, and D. Baleanu, "On fractional filtering versus conventional filtering in economics," Communications in Nonlinear Science and Numerical Simulation, vol. 15, no. 4, pp. 979-986, 2010.

[3] I. Podlubny, Fractional Differential Equations, Academic Press, San Diego, Calif, USA, 1999.

[4] G. Maione and A. Punzi, "Combining differential evolution and particle swarm optimization to tune and realize fractionalorder controllers," Mathematical and Computer Modelling of Dynamical Systems, vol. 19, no. 3, pp. 277-299, 2013.

[5] D. Baleanu, A. K. Golmankhaneh, R. Nigmatullin, and A. K. Golmankhaneh, "Fractional Newtonian mechanics," Central European Journal of Physics, vol. 8, no. 1, pp. 120-125, 2010.

[6] C. Farges, M. Moze, and J. Sabatier, "Pseudo-state feedback stabilization of commensurate fractional order systems," Automatica, vol. 46, no. 10, pp. 1730-1734, 2010.

[7] Rajeev and M. Kushwaha, "Homotopy perturbation method for a limit case Stefan problem governed by fractional diffusion equation," Applied Mathematical Modelling, vol. 37, no. 5, pp. 3589-3599, 2013.

[8] R. Schumer, M. M. Meerschaert, and B. Baeumer, "Fractional advectiondispersion equations for modeling transport at the Earth surface," Journal of Geophysical Research: Earth Surface, vol. 114, no. 4, Article ID F00A07, 2009.

[9] G. Maione, "High-speed digital realizations of fractional operators in the delta domain," IEEE Transactions on Automatic Control, vol. 56, no. 3, pp. 697-702, 2011.

[10] C. Gong, W. Bao, G. Tang, C. Min, and J. Liu, "An efficient iteration method for toeplitz -plus-band triangular systems generated from fractional ordinary differential equation," Mathematical Problems in Engineering, vol. 2014, Article ID 194249, 5 pages, 2014.

[11] J. C. Trigeassou, N. Maamri, J. Sabatier, and A. Oustaloup, "A Lyapunov approach to the stability of fractional differential equations," Signal Processing, vol. 91, no. 3, pp. 437-445, 2011.

[12] J. Sabatier, M. Moze, and C. Farges, "LMI stability conditions for fractional order systems," Computers and Mathematics with Applications, vol. 59, no. 5, pp. 1594-1609, 2010.

[13] G. Maione, "On the Laguerre rational approximation to fractional discrete derivative and integral operators," IEEE Transactions on Automatic Control, vol. 58, no. 6, pp. 1579-1585, 2013.

[14] H. Jiang, F. Liu, I. Turner, and K. Burrage, "Analytical solutions for the multi-term time-fractional diffusion-wave/diffusion equations in a finite domain," Computers and Mathematics with Applications, vol. 64, no. 10, pp. 3377-3388, 2012.

[15] M. Alipour and D. Baleanu, "Approximate analytical solution for nonlinear system of fractional differential equations by BPs operational matrices," Advances in Mathematical Physics, vol. 2013, Article ID 954015, 9 pages, 2013.

[16] S. Saha Ray, "Analytical solution for the space fractional diffusion equation by two-step adomian decomposition method," Communications in Nonlinear Science and Numerical Simulation, vol. 14, no. 4, pp. 1295-1306, 2009.

[17] S. Momani and Z. Odibat, "Comparison between the homotopy perturbation method and the variational iteration method for linear fractional partial differential equations," Computers and Mathematics with Applications, vol. 54, no. 7-8, pp. 910-919, 2007.

[18] S. Chen, F. Liu, and V. Anh, "A novel implicit finite difference method for the one-dimensional fractional percolation equation," Numerical Algorithms, vol. 56, no. 4, pp. 517-535, 2011. 
[19] J. Yan, G.-M. Tan, and N.-H. Sun, "Optimizing parallel Sn sweeps on unstructured grids for multi-core clusters," Journal of Computer Science and Technology, vol. 28, no. 4, pp. 657-670, 2013.

[20] H. Yang and X.-C. Cai, "Parallel fully implicit two-grid methods for distributed control of unsteady incompressible flows," International Journal for Numerical Methods in Fluids, vol. 72, no. 1, pp. 1-21, 2013.

[21] X. Liu, T. Gu, X. Hang, and Z. Sheng, "A parallel version of QMRCGSTAB method for large linear systems in distributed parallel environments," Applied Mathematics and Computation, vol. 172, no. 2, pp. 744-752, 2006.

[22] D. Ming, "A parallel Attainable Region construction method suitable for implementation on a graphics processing unit (GPU)," Computers and Chemical Engineering, vol. 67, pp. 103120, 2014.

[23] X. Liao, L. Xiao, C. Yang, and Y. Lu, "Milkyway-2 supercomputer: system and application," Frontiers of Computer Science, vol. 8, no. 3, pp. 345-356, 2014.

[24] W. Xu, Y. Lu, Q. Li et al., "Hybrid hierarchy storage system in MilkyWay-2 supercomputer," Frontiers of Computer Science, vol. 8, no. 3, pp. 367-377, 2014.

[25] F. Aquotte and A. F. da Silva, "PSIM: a modular particle system on graphics processing unit," IEEE Latin America Transactions, vol. 12, no. 2, pp. 321-329, 2014.

[26] S. J. Pennycook, S. D. Hammond, S. A. Jarvis, and G. R. Mudalige, "Performance analysis of a hybrid MPI/CUDA implementation of the NASLU benchmark," ACM SIGMETRICS Performance Evaluation Review, vol. 38, no. 4, pp. 23-29, 2011.

[27] D. Mu, P. Chen, and L. Wang, "Accelerating the discontinuous Galerkin method for seismic wave propagation simulations using the graphic processing unit (GPU)-single-GPU implementation," Computers and Geosciences, vol. 51, pp. 282-292, 2013.

[28] S. Mauger, G. C. de Verdière, L. Bergé, and S. Skupin, "GPU accelerated fully space and time resolved numerical simulations of self-focusing laser beams in SBS-active media," Journal of Computational Physics, vol. 235, pp. 606-625, 2013.

[29] J. T. Machado, V. Kiryakova, and F. Mainardi, "Recent history of fractional calculus," Communications in Nonlinear Science and Numerical Simulation, vol. 16, no. 3, pp. 1140-1153, 2011.

[30] J. A. Tenreiro Machado, V. Kiryakova, and F. Mainardi, "A poster about the recent history of fractional calculus," Fractional Calculus \& Applied Analysis, vol. 13, no. 3, pp. 329-334, 2010.

[31] A. Loverro, "Fractional calculus: history, definitions and applications for the engineer," Tech. Rep. 46556, Department of Aerospace and Mechanical Engineering, Notre Dame, Ind, USA, 2004.

[32] K. Nishimoto, An Essence of Nishimoto's Fractional Calculus (Calculus in the 21st Century): Integrations and Differentiations of Arbitrary Order, Descartes Press Company, 1991.

[33] M. Cui, "Compact alternating direction implicit method for two-dimensional time fractional diffusion equation," Journal of Computational Physics, vol. 231, no. 6, pp. 2621-2633, 2012.

[34] W. Zhang, X. Cai, and S. Holm, “Time-fractional heat equations and negative absolute temperatures," Computers and Mathematics with Applications, vol. 67, no. 1, pp. 164-171, 2014.

[35] Q. Yang, F. Liu, and I. Turner, "Numerical methods for fractional partial differential equations with Riesz space fractional derivatives," Applied Mathematical Modelling: Simulation and
Computation for Engineering and Environmental Systems, vol. 34, no. 1, pp. 200-218, 2010.

[36] J. Zhao, J. Xiao, and Y. Xu, "A finite element method for the multiterm time-space Riesz fractional advection-diffusion equations in finite domain," Abstract and Applied Analysis, vol. 2013, Article ID 868035, 15 pages, 2013.

[37] Y. Lin and C. Xu, "Finite difference/spectral approximations for the time-fractional diffusion equation," Journal of Computational Physics, vol. 225, no. 2, pp. 1533-1552, 2007.

[38] C. Tadjeran and M. M. Meerschaert, "A second-order accurate numerical method for the two-dimensional fractional diffusion equation," Journal of Computational Physics, vol. 220, no. 2, pp. 813-823, 2007.

[39] N. J. Ford, J. Xiao, and Y. Yan, "Stability of a numerical method for a space-time-fractional telegraph equation," Computational Methods in Applied Mathematics, vol. 12, no. 3, pp. 273-288, 2012.

[40] T. Chang, "Boundary integral operator for the fractional laplace equation in a bounded lipschitz domain," Integral Equations and Operator Theory, vol. 72, no. 3, pp. 345-361, 2012.

[41] G. Jumarie, "Derivation and solutions of some fractional BlackScholes equations in coarse-grained space and time. Application to Merton's optimal portfolio," Computers \& Mathematics with Applications, vol. 59, no. 3, pp. 1142-1164, 2010.

[42] P. Straka, M. M. Meerschaert, R. J. McGough, and Y. Zhou, "Fractional wave equations with attenuation," Fractional Calculus and Applied Analysis, vol. 16, no. 1, pp. 262-272, 2013.

[43] W. Deng, "Finite element method for the space and time fractional Fokker-Planck equation," SIAM Journal on Numerical Analysis, vol. 47, no. 1, pp. 204-226, 2008.

[44] S. Momani and Z. Odibat, "Analytical solution of a timefractional Navier-Stokes equation by Adomian decomposition method," Applied Mathematics and Computation, vol. 177, no. 2, pp. 488-494, 2006.

[45] M. A. Herzallah and D. Baleanu, "Fractional Euler-Lagrange equations revisited," Nonlinear Dynamics, vol. 69, no. 3, pp. 977982, 2012.

[46] A. I. Saichev and G. M. Zaslavsky, "Fractional kinetic equations: solutions and applications," Chaos, vol. 7, no. 4, pp. 753-764, 1997.

[47] T. Wenchang, P. Wenxiao, and X. Mingyu, "A note on unsteady flows of a viscoelastic fluid with the fractional Maxwell model between two parallel plates," International Journal of Non-Linear Mechanics, vol. 38, no. 5, pp. 645-650, 2003.

[48] R. A. El-Nabulsi, “The fractional Boltzmann transport equation," Computers and Mathematics with Applications, vol. 62, no. 3, pp. 1568-1575, 2011.

[49] K. Diethelm, "An investigation of some nonclassical methods for the numerical approximation of Caputo-type fractional derivatives," Numerical Algorithms, vol. 47, no. 4, pp. 361-390, 2008.

[50] K. Diethelm, N. J. Ford, and A. D. Freed, "A predictor-corrector approach for the numerical solution of fractional differential equations," Nonlinear Dynamics, vol. 29, no. 1-4, pp. 3-22, 2002.

[51] M. Cui, "Compact finite difference method for the fractional diffusion equation," Journal of Computational Physics, vol. 228, no. 20, pp. 7792-7804, 2009.

[52] N. J. Ford, J. Xiao, and Y. Yan, "A finite element method for time fractional partial differential equations," Fractional Calculus and Applied Analysis, vol. 14, no. 3, pp. 454-474, 2011. 
[53] X. Zhang, J. Liu, J. Wen, B. Tang, and Y. He, "Analysis for onedimensional time-fractional Tricomi-type equations by LDG methods," Numerical Algorithms, vol. 63, no. 1, pp. 143-164, 2013.

[54] K. Mustapha and W. McLean, "Uniform convergence for a discontinuous Galerkin, time-stepping method applied to a fractional diffusion equation," IMA Journal of Numerical Analysis, vol. 32, no. 3, pp. 906-925, 2012.

[55] Q. Yang, I. Turner, F. Liu, and M. Ilić, "Novel numerical methods for solving the time-space fractional diffusion equation in two dimensions," SIAM Journal on Scientific Computing, vol. 33, no. 3, pp. 1159-1180, 2011.

[56] H. Hejazi, T. Moroney, and F. Liu, "A finite volume method for solving the two-sided time-space fractional advectiondispersion equation," Central European Journal of Physics, vol. 11, no. 10, pp. 1275-1283, 2013.

[57] H. Hejazi, T. Moroney, and F. Liu, "Stability and convergence of a finite volume method for the space fractional advectiondispersion equation," Journal of Computational and Applied Mathematics, vol. 255, pp. 684-697, 2014.

[58] S. Momani, "An explicit and numerical solutions of the fractional KdV equation," Mathematics and Computers in Simulation, vol. 70, no. 2, pp. 110-118, 2005.

[59] B. Tang, C. Jiang, and H. Zhu, "Fractional fourier transform for confluent hypergeometric beams," Physics Letters A, vol. 376, no. 38-39, pp. 2627-2631, 2012.

[60] A. R. Carella and C. A. Dorao, "Least-squares spectral method for the solution of a fractional advection-dispersion equation," Journal of Computational Physics, vol. 232, pp. 33-45, 2013.

[61] A. Shirzadi, L. Ling, and S. Abbasbandy, "Meshless simulations of the two-dimensional fractional-time convection-diffusionreaction equations," Engineering Analysis with Boundary Elements, vol. 36, no. 11, pp. 1522-1527, 2012.

[62] Q. Liu, F. Liu, I. Turner, V. Anh, and Y. T. Gu, "A RBF meshless approach for modeling a fractal mobile/immobile transport model," Applied Mathematics and Computation, vol. 226, pp. 336-347, 2014.

[63] Q. Liu, Y. T. Gu, P. Zhuang, F. Liu, and Y. F. Nie, "An implicit RBF meshless approach for time fractional diffusion equations," Computational Mechanics, vol. 48, no. 1, pp. 1-12, 2011.

[64] R. Garrappa, "A family of Adams exponential integrators for fractional linear systems," Computers and Mathematics with Applications, vol. 66, no. 5, pp. 717-727, 2013.

[65] R. Garrappa and M. Popolizio, "Generalized exponential time differencing methods for fractional order problems," Computers \& Mathematics with Applications, vol. 62, no. 3, pp. 876-890, 2011.

[66] D. Fulger, E. Scalas, and G. Germano, "Monte Carlo simulation of uncoupled continuous-time random walks yielding a stochastic solution of the space-time fractional diffusion equation," Physical Review E, vol. 77, no. 2, Article ID 021122, 2008.

[67] C. Gong, W. Bao, G. Tang, Y. Jiang, and J. Liu, "A parallel algorithm for the two-dimensional time fractional diffusion equation with implicit difference method," The Scientific World Journal, vol. 2014, Article ID 219580, 8 pages, 2014.

[68] J. Q. Murillo and S. B. Yuste, "An explicit difference method for solving fractional diffusion and diffusion-wave equations in the caputo form," Journal of Computational and Nonlinear Dynamics, vol. 6, no. 2, Article ID 021014, 2011.

[69] C. Gong, W. Bao, G. Tang, B. Yang, and J. Liu, "An efficient parallel solution for Caputo fractional reaction-diffusion equation," Journal of Supercomputing, vol. 68, no. 3, pp. 1521-1537, 2014.
[70] C. Tadjeran, M. M. Meerschaert, and H. P. Scheffler, "A secondorder accurate numerical approximation for the fractional diffusion equation," Journal of Computational Physics, vol. 213, no. 1, pp. 205-213, 2006, http://dx.doi.org/10.1016/j.jcp.2005.08.008.

[71] M. M. Meerschaert, D. A. Benson, H.-P. Scheffler, and P. BeckerKern, "Governing equations and solutions of anomalous random walk limits," Physical Review E, vol. 66, no. 6, Article ID 060102, 2002.

[72] R. Metzler and J. Klafter, "The restaurant at the end of the random walk: recent developments in the description of anomalous transport by fractional dynamics," Journal of Physics A: Mathematical and General, vol. 37, no. 31, pp. R161-R208, 2004.

[73] K. S. Miller and B. Ross, An Introduction to the Fractional Calculus and Fractional Differential Equations, John Wiley \& Sons, New York, NY, USA, 1993.

[74] S. Shen, F. Liu, V. Anh, and I. Turner, "The fundamental solution and numerical solution of the Riesz fractional advectiondispersion equation," IMA Journal of Applied Mathematics, vol. 73 , no. 6, pp. 850-872, 2008.

[75] C. Çelik and M. Duman, "Crank-Nicolson method for the fractional diffusion equation with the Riesz fractional derivative," Journal of Computational Physics, vol. 231, no. 4, pp. 1743-1750, 2012.

[76] J. Chen and F. Liu, "Analysis of stability and convergence of numerical approximation for the Riesz fractional reactiondispersion equation," Journal of Xiamen University, vol. 45, no. 4, pp. 466-469, 2006 (Chinese).

[77] C. Gong, W. Bao, and G. Tang, "A parallel algorithm for the Riesz fractional reaction-diffusion equation with explicit finite difference method," Fractional Calculus and Applied Analysis, vol. 16, no. 3, pp. 654-669, 2013.

[78] A. Yildirim and H. Koçak, "Homotopy perturbation method for solving the space-time fractional advection-dispersion equation," Advances in Water Resources, vol. 32, no. 12, pp. 1711-1716, 2009.

[79] S. Shen, F. Liu, and V. Anh, "Numerical approximations and solution techniques for the space-time Riesz-Caputo fractional advection-diffusion equation," Numerical Algorithms, vol. 56, no. 3, pp. 383-403, 2011.

[80] F. Liu, P. Zhuang, V. Anh, I. Turner, and K. Burrage, "Stability and convergence of the difference methods for the space-time fractional advection-diffusion equation," Applied Mathematics and Computation, vol. 191, no. 1, pp. 12-20, 2007.

[81] C.-M. Chen and F. Liu, "A numerical approximation method for solving a three-dimensional space Galilei invariant fractional advection-diffusion equation," Journal of Applied Mathematics and Computing, vol. 30, no. 1-2, pp. 219-236, 2009.

[82] K. Mustapha, "An implicit finite-difference time-stepping method for a sub-diffusion equation, with spatial discretization by finite elements," IMA Journal of Numerical Analysis, vol. 31, no. 2, pp. 719-739, 2011.

[83] J. Huang, N. Nie, and Y. Tang, "A second order finite differencespectral method for space fractional diffusion equations," Science China Mathematics, vol. 57, no. 6, pp. 1303-1317, 2014.

[84] P. Zhuang, F. Liu, V. Anh, and I. Turner, "Numerical methods for the variable-order fractional advection-diffusion equation with a nonlinear source term," SIAM Journal on Numerical Analysis, vol. 47, no. 3, pp. 1760-1781, 2009.

[85] M. Cui, "Convergence analysis of high-order compact alternating direction implicit schemes for the two-dimensional time fractional diffusion equation," Numerical Algorithms, vol. 62, no. 3, pp. 383-409, 2013. 
[86] H. Wang and T. S. Basu, "A fast finite difference method for two-dimensional space-fractional diffusion equations," SIAM Journal on Scientific Computing, vol. 34, no. 5, pp. A2444A2458, 2012.

[87] P. Zhuang and F. Liu, "Finite difference approximation for two-dimensional time fractional diffusion equation," Journal of Algorithms \& Computational Technology, vol. 1, no. 1, pp. 1-15, 2007.

[88] H. Sun, W. Chen, and Y. Chen, "Variable-order fractional differential operators in anomalous diffusion modeling," Physica A: Statistical Mechanics and Its Applications, vol. 388, no. 21, pp. 4586-4592, 2009.

[89] X. Liao, "MilkyWay-2: back to the world Top 1," Frontiers of Computer Science, vol. 8, no. 3, pp. 343-344, 2014.

[90] S. J. Pennycook, S. D. Hammond, G. R. Mudalige, S. A. Wright, and S. A. Jarvis, "On the acceleration of wavefront applications using distributed many-core architectures," Computer Journal, vol. 55, no. 2, pp. 138-153, 2012.

[91] Q. Xu, G.-L. Yu, K. Wang, and J.-L. Sun, "Research on gpuaccelerated algorithm in 3d finite difference neutro $\mathrm{n}$ diffusion calculation method," Nuclear Science and Techniques, vol. 25, no. 1, Article ID 010501, 2014.

[92] M. Rosa, J. S. Warsa, and M. Perks, "A cellwise block-gaussseidel iterative method for multigroup $S_{N}$ transport on a hybrid parallel computer architecture," Nuclear Science and Engineering, vol. 174, no. 3, pp. 209-226, 2013.

[93] A. K. Jha, M. A. Kupinski, H. H. Barrett, E. Clarkson, and J. H. Hartman, "Three-dimensional Neumann-series approach to model light transport in nonuniform media," Journal of the Optical Society of America A, vol. 29, no. 9, pp. 1885-1899, 2012.

[94] C. Xu, X. Deng, L. Zhang et al., "Parallelizing a high-order CFD software for 3D, multi-block, structural grids on the TianHe-1A supercomputer," in Supercomputing, vol. 7905 of Lecture Notes in Computer Science, Springer, Berlin, Germany, 2013.

[95] Y.-X. Wang, L.-L. Zhang, W. Liu et al., "Efficient parallel implementation of large scale 3D structured grid CFD applications on the Tianhe-1A supercomputer," Computers \& Fluids, vol. 80, no. 1, pp. 244-250, 2013.

[96] Q. Wu, C. Yang, T. Tang, and L. Xiao, "Exploiting hierarchy parallelism for molecular dynamics on a petascale heterogeneous system," Journal of Parallel and Distributed Computing, vol. 73, no. 12, pp. 1592-1604, 2013.

[97] F. Chen and J. Shen, "A GPU parallelized spectral method for elliptic equations in rectangular domains," Journal of Computational Physics, vol. 250, pp. 555-564, 2013.

[98] Z. Mo, A. Zhang, X. Cao et al., "JASMIN: a parallel software infrastructure for scientific computing," Frontiers of Computer Science in China, vol. 4, no. 4, pp. 480-488, 2010.

[99] C. Xu, X. Deng, L. Zhang et al., "Collaborating CPU and GPU for large scale high-order CFD for simulations with complex grids on the tianhe-1A supercom puter," Journal of Computational Physics, vol. 278, pp. 275-297, 2014.

[100] R. Chen, Y. Wu, Z. Yan, Y. Zhao, and X.-C. Cai, "A parallel domain decomposition method for 3D unsteady incompressible flows at high reynolds number," Journal of Scientific Computing, vol. 58, no. 2, pp. 275-289, 2014.

[101] H. Yang, C. Yang, and X.-C. Cai, "Parallel domain decomposition methods with mixed order discretization for fully implicit solution of tracer transport problems on the cubed-sphere," Journal of Scientific Computing, 2014.
[102] C. Yang, J. Cao, and X. C. Cai, "A fully implicit domain decomposition algorithm for shallow water equations on the cubed-sphere," SIAM Journal on Scientific Computing, vol. 32, no. 1, pp. 418-438, 2010.

[103] C. Gong, W. Bao, G. Tang, Y. Jiang, and J. Liu, "A domain decomposition method for time fractional reaction-diffusion equation," The Scientific World Journal, vol. 2014, Article ID 681707, 5 pages, 2014.

[104] K. Diethelm, "An efficient parallel algorithm for the numerical solution of fractional differential equations," Fractional Calculus and Applied Analysis, vol. 14, no. 3, pp. 475-490, 2011.

[105] C. Gong, J. Liu, L. Chi, H. Huang, J. Fang, and Z. Gong, "GPU accelerated simulations of $3 \mathrm{D}$ deterministic particle transport using discrete ordinates method," Journal of Computational Physics, vol. 230, no. 15, pp. 6010-6022, 2011.

[106] C. Gong, J. Liu, Z. Gong, J. Qin, and J. Xie, "Optimizing Sweep3D for graphic processor unit," in Algorithms and Architectures for Parallel Processing, C.-H. Hsu, L. Yang, J. Park, and S.-S. Yeo, Eds., vol. 6081 of Lecture Notes in Computer Science, pp. 416-426, Springer, Berlin, Germany, 2010.

[107] X. Gan, C. Liu, Z. Wang et al., "Accelerating GOR algorithm using CUDA," Applied Mathematics and Information Sciences, vol. 7, no. 2, pp. 563-567, 2013.

[108] C. Gong, J. Liu, H. Huang, and Z. Gong, "Particle transport with unstructured grid on GPU," Computer Physics Communications, vol. 183, no. 3, pp. 588-593, 2012.

[109] X. Gan, Z. Wang, L. Shen, and Q. Zhu, "Data layout pruning on GPU," Applied Mathematics \& Information Sciences, vol. 5, no. 2, pp. 129-138, 2011.

[110] W. Zhang, W. Wei, and X. Cai, "Performance modeling of serial and parallel implementations of the fractional AdamsBashforth-Moulton method," Fractional Calculus and Applied Analysis, vol. 17, no. 3, pp. 617-637, 2014.

[111] J. Liu, C. Gong, W. Bao, G. Tang, and Y. Jiang, "Solving the Caputo fractional reaction-diffusion equation on GPU," Discrete Dynamics in Nature and Society, vol. 2014, Article ID 820162, 2014.

[112] Y. Xu and Z. He, "The short memory principle for solving Abel differential equation of fractional order," Computers and Mathematics with Applications, vol. 62, no. 12, pp. 4796-4805, 2011.

[113] W. Deng, "Short memory principle and a predictor-corrector approach for fractional differential equations," Journal of Computational and Applied Mathematics, vol. 206, no. 1, pp. 174-188, 2007.

[114] N. J. Ford and A. C. Simpson, "The numerical solution of fractional differential equations: speed versus accuracy," Numerical Algorithms, vol. 26, no. 4, pp. 333-346, 2001.

[115] H.-L. Cao, X. Li, Z.-H. Deng, and Y. Qin, "Control-oriented fast numerical approaches of fractional-order models," Control Theory and Applications, vol. 28, no. 5, pp. 715-721, 2011 (Chinese).

[116] H. Wang, K. Wang, and T. Sircar, "A direct $O\left(N \log ^{2} N\right)$ finite difference method for fractional diffusion equations," Journal of Computational Physics, vol. 229, no. 21, pp. 8095-8104, 2010.

[117] H. Wang and K. Wang, "An $O\left(N \log ^{2} N\right)$ alternating-direction finite difference method for two-dimensional fractional diffusion equations," Journal of Computational Physics, vol. 230, no. 21, pp. 7830-7839, 2011.

[118] H. Wang and N. Du, "A superfast-preconditioned iterative method for steady-state space-fractional diffusion equations," Journal of Computational Physics, vol. 240, pp. 49-57, 2013. 
[119] H.-K. Pang and H.-W. Sun, "Multigrid method for fractional diffusion equations," Journal of Computational Physics, vol. 231, no. 2, pp. 693-703, 2012.

[120] Z. Zhou and H. Wu, "Finite element multigrid method for the boundary value problem of fractional advection dispersion equation," Journal of Applied Mathematics, vol. 2013, Article ID 385403, 8 pages, 2013.

[121] S.-L. Lei and H.-W. Sun, "A circulant preconditioner for fractional diffusion equations," Journal of Computational Physics, vol. 242, pp. 715-725, 2013.

[122] T. Moroney and Q. Yang, "A banded preconditioner for the twosided, nonlinear space-fractional diffusion equation," Computers and Mathematics with Applications, vol. 66, no. 5, pp. 659667, 2013.

[123] T. Moroney and Q. Yang, "Efficient solution of two-sided nonlinear space-fractional diffusion equations using fast poisson preconditioners," Journal of Computational Physics, vol. 246, pp. 304-317, 2013.

[124] J. A. Tenreiro Machado, "Fractional derivatives: probability interpretation and frequency response of rational approximations," Communications in Nonlinear Science and Numerical Simulation, vol. 14, no. 9-10, pp. 3492-3497, 2009.

[125] F.-R. Lin, S.-W. Yang, and X.-Q. Jin, "Preconditioned iterative methods for fractional diffusion equation," Journal of Computational Physics, vol. 256, pp. 109-117, 2014.

[126] W. Deng, S. Du, and Y. Wu, "High order finite difference WENO schemes for fractional differential equations," Applied Mathematics Letters, vol. 26, no. 3, pp. 362-366, 2013.

[127] H. Zhou, W. Tian, and W. Deng, "Quasi-compact finite difference schemes for space fractional diffusion equations," Journal of Scientific Computing, vol. 56, no. 1, pp. 45-66, 2013.

[128] C. Lubich, "Discretized fractional calculus," SIAM Journal on Mathematical Analysis, vol. 17, no. 3, pp. 704-719, 1986. 


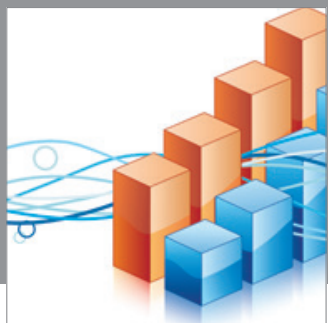

Advances in

Operations Research

mansans

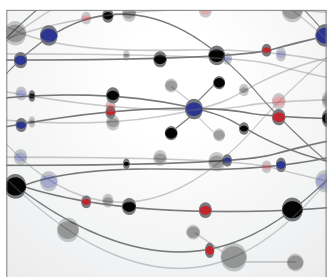

The Scientific World Journal
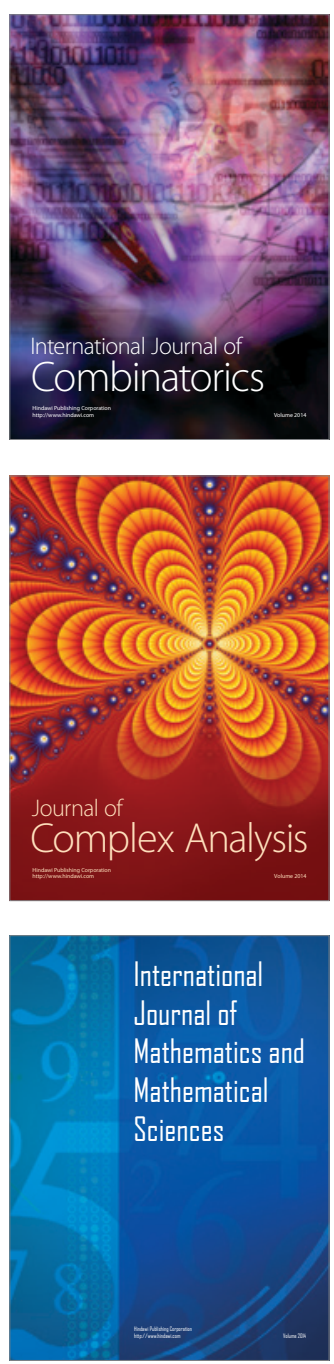
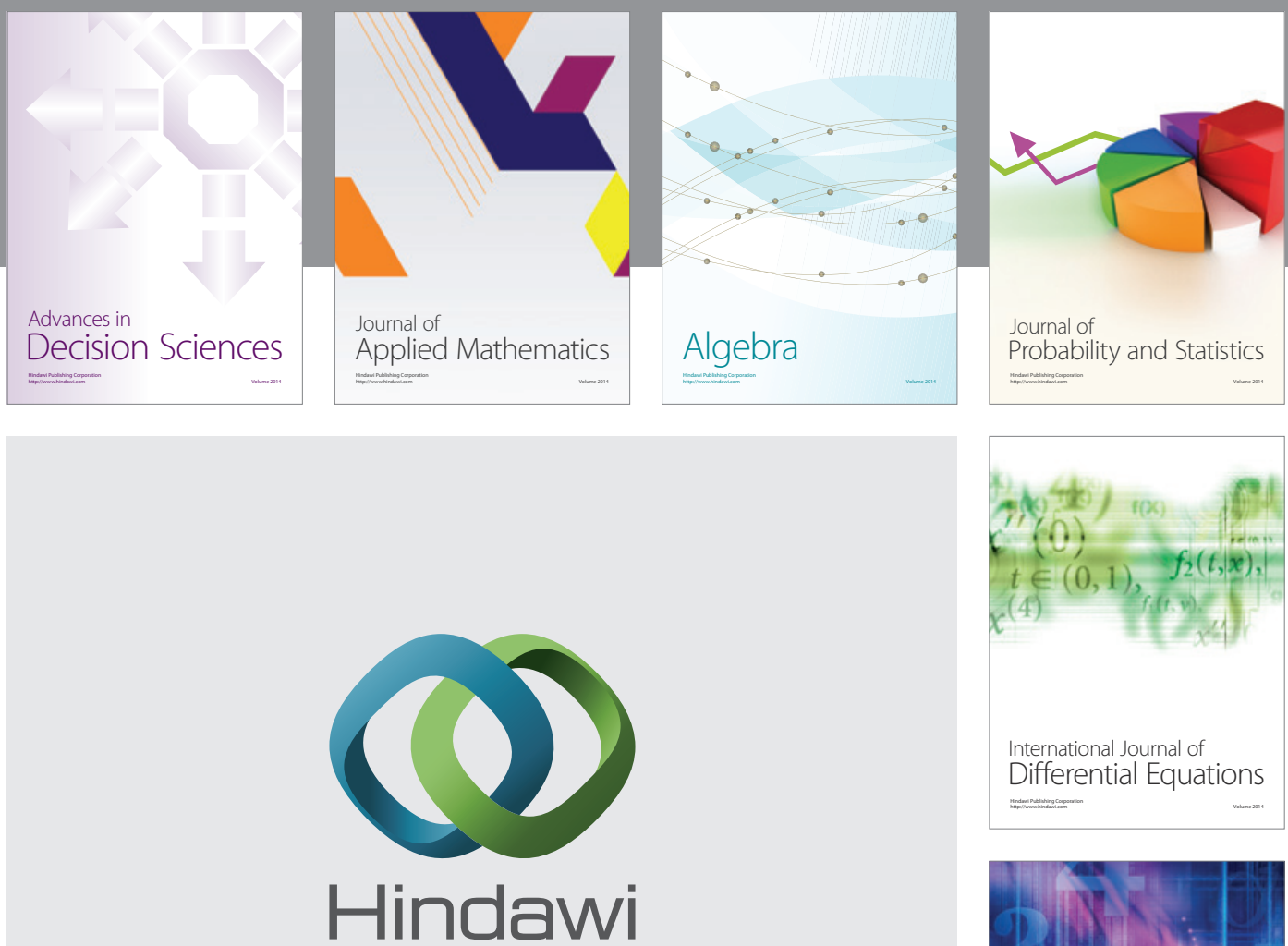

Submit your manuscripts at http://www.hindawi.com
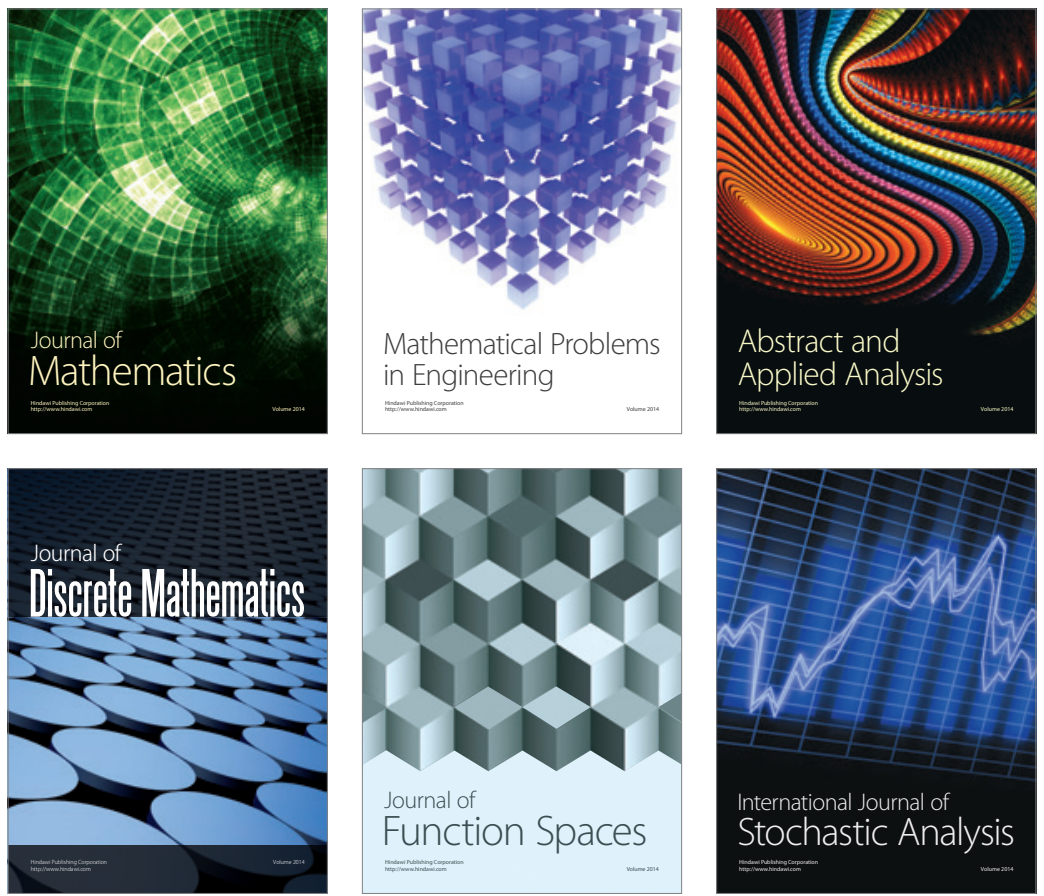

Journal of

Function Spaces

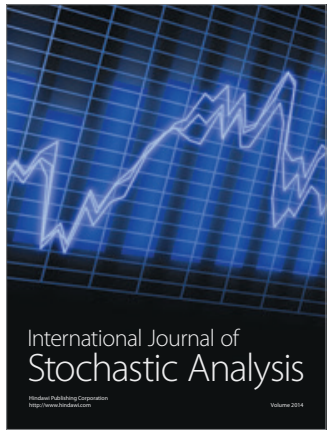

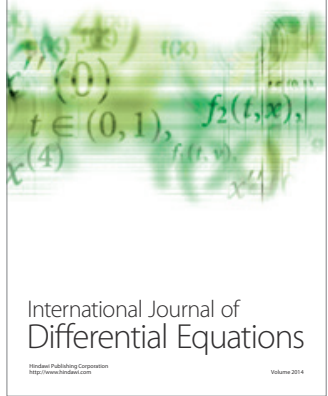
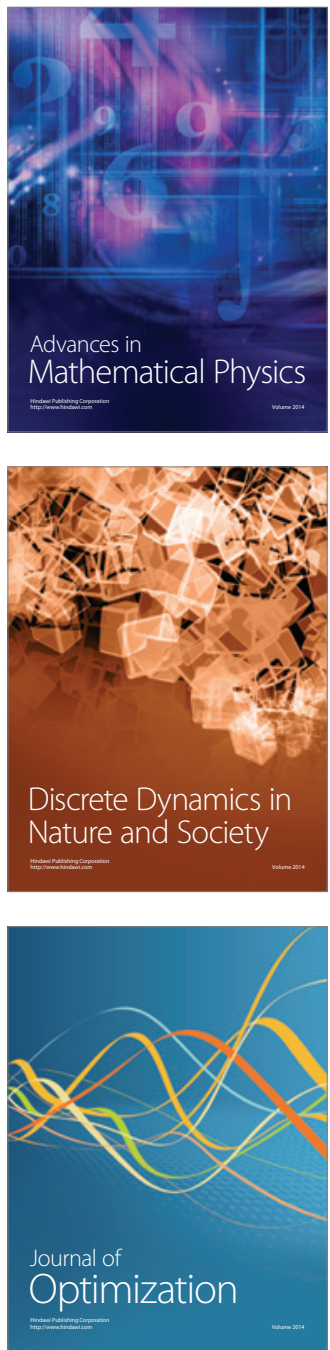